\title{
New observations of the pulsating PMS star V351 Ori
}

\author{
M. Marconi ${ }^{1}$, V. Ripepi ${ }^{1}$, S. Bernabei ${ }^{2,3}$, F. Palla ${ }^{4}$, J. M. Alcalà ${ }^{1}$, E. Covino ${ }^{1}$, and L. Terranegra ${ }^{1}$ \\ 1 Osservatorio Astronomico di Capodimonte, Via Moiariello 16, 80131 Napoli, Italy \\ 2 Osservatorio Astronomico di Bologna, Via Ranzani 1, 40127 Bologna, Italy \\ 3 Dipartimento de Astrofísica, Universidad de La Laguna, Avda. Astrofisico F. Sànchez sn, \\ 30071 La Laguna, Spain \\ ${ }^{4}$ Osservatorio Astrofisico di Arcetri, Largo E. Fermi, 5, 50125 Firenze, Italy
}

Received 23 March 2001 / Accepted 17 April 2001

\begin{abstract}
We present new precise photoelectric observations of the Pre-Main-Sequence $\delta$ Scuti star, V351 Ori. These new data show that V351 Ori pulsates in a mixture of several radial modes (at least four). The comparison between observations and detailed pulsational models, allows us to provide independent constraints on the mass and luminosity of the star. The predicted distance is $210 \mathrm{pc}$, indicating that V351 Ori is much closer than the Orion star forming region. With an inferred mass of $\sim 1.8 M_{\odot}$ and an uncertain evolutionary stage, V351 Ori represents an excellent candidate for future asteroseismological studies that will assess whether it is a young PMS star ( $\sim 6 \mathrm{Myr})$ or an evolved object ( 1 Gyr) leaving the main-sequence.
\end{abstract}

Key words. stars: variables: $\delta$ Scuti - stars: oscillations - stars: fundamental parameters

\section{Introduction}

The existence of pulsating stars among intermediate mass $\left(1.5 \leq M_{\odot} \leq 5\right)$ Herbig Ae stars was originally suggested by Breger (1972). The later observations of $\delta$ Scuti like pulsation in the Herbig Ae stars HR 5999 (Kurtz \& Marang 1995) and HD 104237 (Donati et al. 1997) stimulated a theoretical investigation of the instability strip for Pre-Main-Sequence (PMS) stars (Marconi \& Palla 1998, hereinafter MP98). These computations, based on nonlinear convective hydrodynamical models, allowed to define for the first time the topology of the instability region of the first three radial modes. At the same time MP98 identified possible candidates for pulsational variability among known Herbig Ae/Be stars located within or close to the predicted instability strip boundaries.

As a result, several photometric investigations were devoted to the analysis of these young objects. Marconi et al. (1900, hereinafter M00) discovered a $\delta$ Scuti type variability in the PMS Herbig Ae stars HD 35929 and V351 Ori. At the same time Pigulski et al. (2000a,b) announced the discovery of $\delta$ Scuti pulsation in the Herbig Ae stars BL 50 and HP 57 belonging to the open cluster NGC 6823. More recently, Kurtz \& Müller (2001) have identified a new PMS $\delta$ Scuti star, namely HD 142666 , with a pulsation period of $67 \mathrm{~min}$.

Send offprint requests to: V. Ripepi,

e-mail: ripepi@na.astro.it
The comparison between the observed pulsation properties of PMS stars and the predictions of non-linear hydrodynamical model provides important constraints on the stellar mass and evolutionary state of Herbig Ae stars (see MP98 and M00). As a preliminary application of this kind of analysis, M00 have used the periods of V351 Ori and HD 35929 to identify their pulsation modes and to obtain an independent estimate of the distance. However, the initial observations were limited by the poor sampling and by the small number of phase points, so that only the $\delta$ Scuti-type pulsation was detected, with just one period for each star. The data were not rich enough for the identification of other possible periodicities. This basic limitation has stimulated a new program dedicated to the observations of V351 Ori and HD 35929, plus other previously identified candidates (see M00).

In this paper, we present new high quality photometric observations of V351 Ori. In Sect. 2, we describe the observations and data analysis, while in Sect. 3 we show the frequency analysis. The comparison with theoretical models is described in Sect. 4, and we conclude with a discussion of the evolutionary status of V351 Ori.

\section{Observations and data reduction}

The observations were carried out with the $152 \mathrm{~cm}$ "Cassini" telescope, located in Loiano, Italy. The telescope was equipped with a three channel photometer (called TTCP) which is designed to collect simultaneously the 


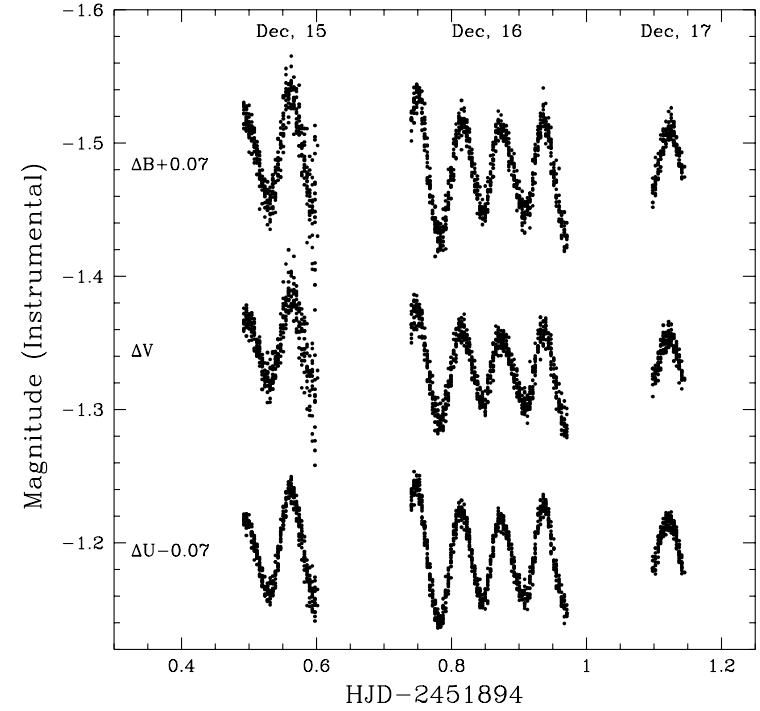

Fig. 1. $U B V$ photometry of V351 Ori. For clarity, the $B$ and $U$ data have been shifted by $\pm 0.07 \mathrm{mag}$. Also, the HJD for the second and third night are shifted by -0.65 day and -1.3 day, respectively.

signal from the target, a comparison star and the sky. The instrument is also capable of fast filter changes $(0.1 \mathrm{~s})^{1}$.

Observations of V351 Ori were obtained during three nights from December 15 to 17, 2000. Because of poor seeing conditions, the aperture used for all the channels was 17 arcsec. We observed in the $U B V$ Johnson system, but we were not able to calibrate the data since no night was photometric. However, this is not a critical point, since the frequency analysis does not require a photometric calibration. Since both V351 Ori and the comparison star, HD 290826, are bright stars, the exposure times were short: 18,3 , and $4 \mathrm{~s}$ in $U B V$, respectively. The resulting photometry is presented in Fig. 1. We note that the light variation is present in all the three filters with a maximum amplitude of $\sim 0.12 \mathrm{mag}$ in the $U$ and $B$ filters. Inspection of the figure also reveals the presence of multi-periodic oscillations. Note also that the scattered data points present at the end of Dec. 15 (see Fig. 1) are due to the worsening of observing conditions and were not included in the analysis.

\section{Frequency analysis}

The frequency analysis was performed independently for each filter, using the program Period98 (http://www.astro.univie.ac.at/ dsn/). We have identified 6 periodicities, whose corresponding frequencies are listed in Table 1. To illustrate the procedure, Fig. 2 shows the frequency analysis step by step in the $U$-filter. The derived frequencies, $f 1$ to $f 6$, have been selected in order of decreasing amplitude.

Inspection of Table 1 and Fig. 2 reveals that all the frequencies are well separated, apart for $f 3$ which is close

${ }^{1}$ A detailed description of TTCP can be found at: http://www.na.astro.it/ silvotti/ttcp
Table 1. Observed frequencies of V351 Ori. Frequencies $f 1$ to $f 6$ are ordered by decreasing amplitude. The error on the individual frequencies is of the order of $0.23 \mathrm{~d}^{-1}$.

\begin{tabular}{ccccc}
\hline \hline Frequency & $\begin{array}{c}U \\
\left(\mathrm{~d}^{-1}\right)\end{array}$ & $\begin{array}{c}B \\
\left(\mathrm{~d}^{-1}\right)\end{array}$ & $\begin{array}{c}V \\
\left(\mathrm{~d}^{-1}\right)\end{array}$ & $\begin{array}{c}\text { Average } \\
\left(\mathrm{d}^{-1}\right)\end{array}$ \\
\hline$f_{1}$ & 15.59 & 15.39 & 15.51 & $15.49 \pm 0.24$ \\
$f_{2}$ & 11.89 & 11.92 & 11.86 & $11.89 \pm 0.23$ \\
$f_{3}$ & 15.85 & 17.27 & 15.69 & $16.27 \pm 0.74$ \\
$f_{4}$ & 31.26 & 32.40 & 30.32 & $31.33 \pm 0.88$ \\
$f_{5}$ & 8.32 & 6.20 & 6.19 & $6.90 \pm 1.03$ \\
$f_{6}$ & 27.08 & 27.14 & 26.09 & $26.77 \pm 0.53$ \\
\hline
\end{tabular}

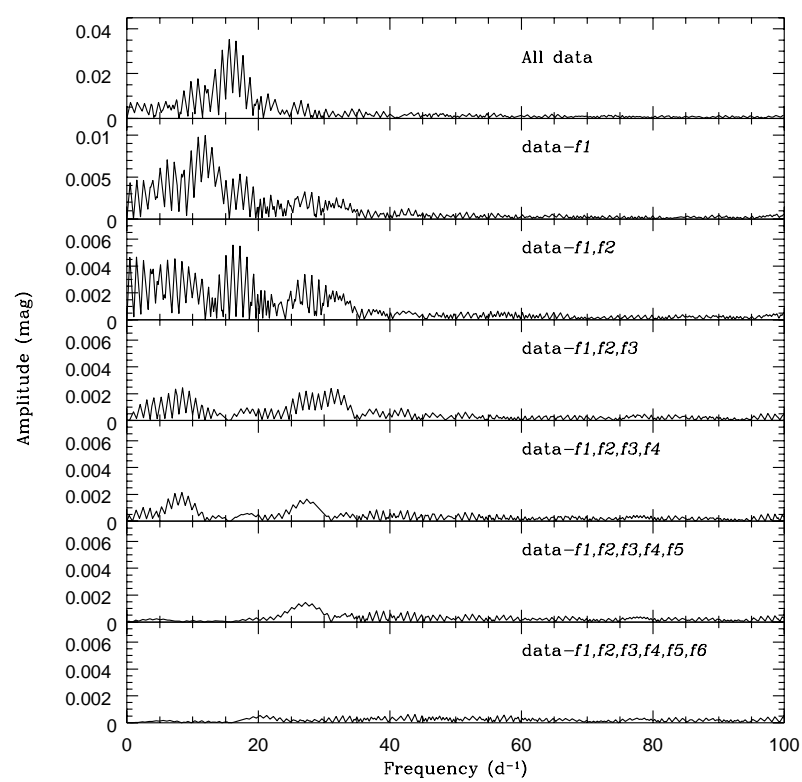

Fig. 2. Example of the frequency analysis in the $U$ filter. The top panel shows the Fourier Transform (FT) for all the data. The other panels display the FT after the successive subtraction of the frequencies $f 1$ to $f 6$.

to $f 1$. Although our data seem to indicate that $f 3$ is real, further observations are needed in order to confirm its identification.

The final result of the frequency analysis is shown in Fig. 3 where we display the observations in the $U$ filter for the night of Dec. 16, 2000. We have superposed to the data points the fit obtained using the six frequencies reported in the first column of Table 1. A similar analysis has been carried out for the $B$ and $V$ filters. The resulting frequencies are also listed in the last column of Table 1. They represent the average values that will be used in the next section to constrain the pulsational models.

\section{Comparison with theory}

In our previous analysis of V351 Ori (see M00), we used the period results to obtain a first estimate of its luminosity, distance, and evolutionary state. At the time, we were not able to discriminate between the distance of the Orion Nebula $(d=450 \mathrm{pc})$ and the significantly smaller 


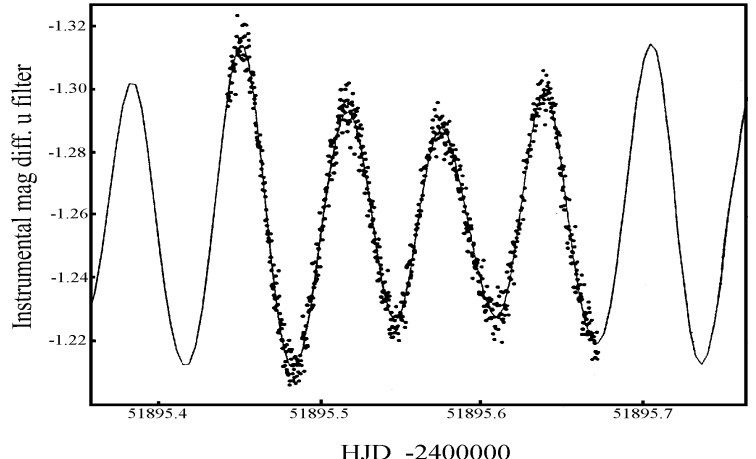

Fig. 3. Dots: data in the $U$ filter for the night Dec. 16, 2000. Solid line: fit to the data obtained using the six frequencies reported in the first row of Table 1.

value measured by Hipparcos $(d=210 \mathrm{pc}$, van den Ancker et al. 1998). The observations presented in this letter are richer and more detailed, allowing us to provide further constraints on the intrinsic stellar parameters and evolutionary properties.

We have now identified at least 4 frequencies that indicate that V351 Ori is pulsating in a mixture of modes. We have tried to reproduce the observed periods with linear non-adiabatic pulsation models of PMS stars (e.g., MP98). We have succeeded in matching the frequencies $f 1, f 2, f 4$ and $f 6$ with a single model with mass $M_{*}=1.8 M_{\odot}$, luminosity $L_{*}=13.87 L_{\odot}$, and effective temperature $T_{\text {eff }}=7350 \mathrm{~K}$. Accordingly, the star should be pulsating in at least four radial modes: the fundamental mode with period $P=0.0841 \mathrm{~d}$ (or $f=11.89 \mathrm{~d}^{-1}$, corresponding to $f 2$ ), the first overtone with $P=0.0647 \mathrm{~d}$ (or $f=15.46 \mathrm{~d}^{-1}$, corresponding to $f 1$ ), the fourth overtone with $P=0.0368 \mathrm{~d}$ ( or $f=27.17 \mathrm{~d}^{-1}$, similar to $f 6$ ), and the fifth overtone with $P=0.0323 \mathrm{~d}$ ( or $f=30.96 \mathrm{~d}^{-1}$, close to $f 4$ ). To provide an estimate of the accuracy of the predicted stellar parameters, we have varied the mass, luminosity and effective temperature, until the resulting frequencies deviate from the observed ones by more than the associated error (see Table 1). As a result we have found that the accuracy is $4 \%$ in mass, $2 \%$ in luminosity and $<1 \%$ in effective temperature. Considering the largest sources of uncertainty, the range of acceptable masses is between 1.73 and $1.87 M_{\odot}$.

The position of the best fit model in the $\mathrm{H}-\mathrm{R}$ diagram is shown in Fig. 4. In the figure we also display the PMS instability strip, and the PMS (Palla \& Stahler 1993) and post-MS (Castellani et al. 1999) evolutionary tracks are shown, for $1.8 M_{\odot}$ and $2.5 M_{\odot}$, as solid and dashed lines, respectively. The dotted box indicates the luminosity range corresponding to the empirical distance determinations available in the literature, with the two triangles marking lower and upper limits. The lower value corresponds to the Hipparcos distance of 210 pc, whereas the upper limit assumes that V351 Ori is located in the Orion star forming region. The width of the box corresponds to an uncertainty of $\pm 0.01 \mathrm{dex}$ in $\log T_{\text {eff }}$, around the empirical mean value $\log T_{\text {eff }} \simeq 3.88$ (van den Ancker et al. 1998).

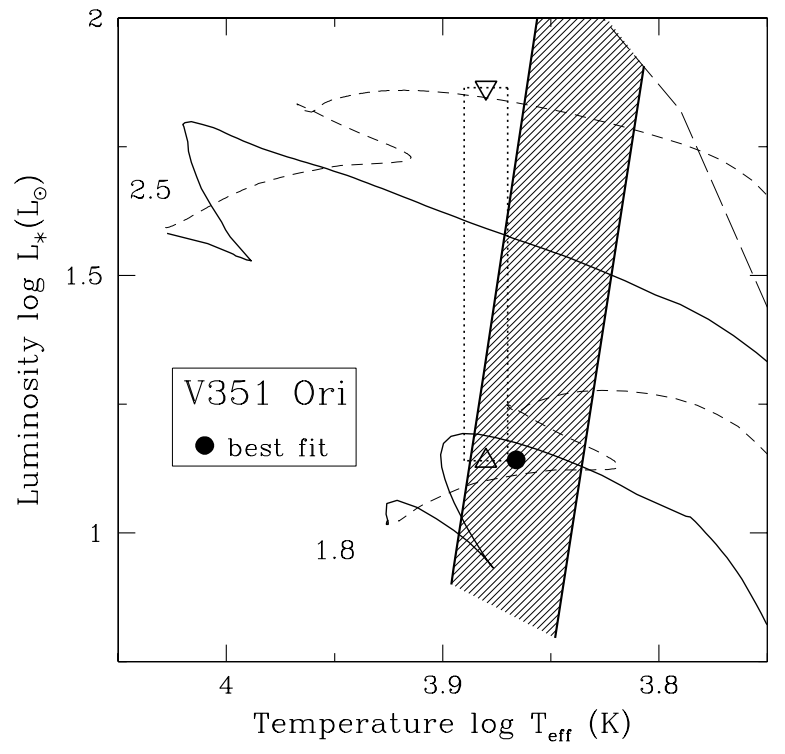

Fig. 4. The position of V351 Ori in the H-R diagram, according to the empirical estimates of spectral type and distance (dotted box) found in the literature. The shaded region is the instability strip predicted by MP98. The PMS and post-MS evolutionary tracks are shown for $1.8 M_{\odot}$ and $2.5 M_{\odot}$ (solid and dashed lines, respectively). The birthline is displayed by the long-dashed line. Finally, the filled circle marks the position of our best fit model (see text).

Our best fit model does not reproduce the frequencies $f 3$ and $f 5$. As already noted, $f 3$ is very close to $f 1$ and consistent within the errors with the first overtone period of the model. Moreover, the period corresponding to $f 5$ is consistent with twice the fundamental period and with twice the first overtone period. This occurrence is not well understood on the basis of present data. If confirmed in the future by more precise observations, this frequency might indicate the occurrence of non-radial modes in addition to the radial ones.

None of the observed periods can be reproduced by models of stars of higher mass. If we take the solution consistent with the location in the Orion region (a PMS star of $\sim 3 M_{\odot}$ ), the predicted periods would be significantly longer $(P \simeq 0.23 \mathrm{~d}$ for the fundamental mode). For the fifth overtone mode we would find a period consistent with the frequency $f 1$ but the spacing with the higher frequencies cannot be reproduced. Moreover, radial modes higher than the fifth are rather unlikely.

\section{The evolutionary status of V351 Ori}

As clearly shown in Fig. 4, the predicted luminosity of V351 Ori corresponds to the lower limit of the empirical distance range. Thus, the pulsation analysis confirms the distance provided by the Hipparcos catalogue, removing the luminosity degeneracy previously found from the determination of a single frequency (M00). However, a smaller distance does not allow us to exclude the possibility that V351 Ori is an older star of about the same mass. According to Fig. 4, the post-MS track of a $1.8 M_{\odot}$ 
star intersects the corresponding PMS track within the instability strip, close to the location of V351 Ori. Since the pulsational properties mainly depend on the surface structure of the stars which is basically the same for pre- and post-MS stars, one does not expect a clear signature that would allow to distinguish between a young $(\sim 6.5 \mathrm{Myr})$ and an evolved ( 1 Gyr) star.

What is the present understanding of the evolutionary status of V351 Ori? The real nature of the star is still debated in the literature. Its strong photometric variations are attributed to extinction by circumstellar dust clouds and have been used to argue for a relative youth of the star. Such variations, in fact, have been observed in other Herbig stars, e.g. BN Ori (Shevchenko et al. 1997), and should signal the transition from an active PMS phase to the arrival at the main sequence. Other signs of a young age include the presence of $\mathrm{H} \alpha$ emission with a strong inverse P Cygni profile (van den Ancker et al. 1996), the SI $\lambda 1296 \AA$ and OI $\lambda 1304 \AA$ UV lines (Valenti et al. 2000), and broad infrared excess (van den Ancker et al. 1997). All these are indices of accretion from and interaction with a circumstellar disk/shell.

The possibility that V351 Ori is an old star comes from the lack of a reflection nebula and of association with an obscured region (i.e. the criteria for classification as a Herbig Ae star) and a space velocity which indicates that the star is approaching the Orion star forming region, but is not kinematically linked to it. Spectral analysis of hydrogen absorption lines also show peculiarities which led Koval'chuck \& Pugach (1998) to derive a very low hydrogen abundance, with no metals. However, we believe that such an anomalous chemical composition is inconsistent with the observed pulsational properties of the star. In fact, a reduction of hydrogen and the concurrent increase of the helium abundance produce models with periods much longer than the observed ones. We believe that the interpretation of the chemical anomalies of V351 Ori in the framework of $\lambda$ Boötis stars suggested by Gray \& Corbally (1998) is more consistent with the observed pulsations. From a statistical analysis of a large number of objects, Gray \& Corbally conclude that the incidence of young and old $\lambda$ Boötis stars is approximately the same, and represents about $3 \%$ of all A stars. Thus, the association of V351 Ori with this class of variable stars is not per se an age discriminator.

From the theoretical viewpoint, while radial modes cannot help distinguishing between the two age estimates, non-radial modes certainly can. As recently shown by Suran et al. (2001), the internal structure of a $1.8 M_{\odot}$ pre-MS star differs substantially from the post-MS case.
In particular, the presence of an expanding convective core and of mean molecular weight gradients in the evolved star induce the excitation of non-radial (both gravity and pressure) modes. For the $1.8 M_{\odot}$ star, low frequency $g$-modes in the range $4-10$ are the most sensitive to the detailed structure of the core. Suran et al. (2001) conclude that the expected amplitudes are large enough that they could be detected both from the ground (as for classical $\delta$ Scuti stars), and from space using, e.g., the COROT satellite. We thus conclude that V351 Ori with its inferred mass of $\sim 1.8 M_{\odot}$ represents an excellent candidate for asteroseismological studies.

Acknowledgements. It is a pleasure to thank Roberto Silvotti for his valuable help with the TTCP photometer. We also wish to thank the personnel of the Loiano Observatory for their kind support. Finally, we thank our referee, Mario van den Ancker for useful comments and a careful review of the paper.

\section{References}

Breger, M. 1972, ApJ, 171, 539

Castellani, V., Degl'Innocenti, S., \& Marconi, M. 1999, MNRAS, 303, 265

Donati, J.-F., Semel, M., Carter, B. D., Rees, D. E., \& Cameron, A. C. 1997, MNRAS, 291, 658

Gray, R. O., \& Corbally, C. J. 1998, AJ, 116, 2530

Koval'chuk, G. U., \& Pugach, A. F. 1998, AstL, 24, 106

Kurtz, D. W., \& Marang, F. 1995, MNRAS, 276, 191

Kurtz, D. W., \& Müller, M. 2001, MNRAS, in press

Marconi, M., \& Palla, F. 1998, ApJ, 507, L141 (MP98)

Marconi, M., Ripepi, V., Alcalà, J. M., et al. 2000, A\&A, 355, L35 (M00)

Palla, F., \& Stahler, S. W. 1993, ApJ, 418, 414

Pigulski, A., Kołaczkowski, Z., \& Kopacki, G. 2000a, AcA, 50, 113

Pigulski, A., Kołaczkowski, Z., \& Kopacki, G. 2000b, in IAU Colloq. 176, The Impact of Large-Scale Surveys on Pulsating Star Research, ed. L. Szabados, \& D. Kurtz (San Francisco: ASP), in press

Shevchenko, V. S., Ezhkova, O., Tjin A Djie, H. R. E., et al. 1997, A\&AS, 124, 33

Suran, M., Goupil, M., Baglin, A., Lebreton, Y., \& Catala, C. 2001, A\&A, in press

Valenti, J. A., Johns-Krull, C. M., \& Linsky, J. L. 2000, ApJS, 129,399

van den Ancker, M. E., Thé, P. S., \& de Winter, D. 1996, A\&A, 309, 809

van den Ancker, M. E., Thé, P. S., Feinstein, A., et al. 1997, A\&AS, 123, 63

van den Ancker, M. E., de Winter, D., \& Tjin A Djie, H. R. E. 1998, A\&A, 330, 145 